Document downloaded from:

http://hdl.handle.net/10251/37774

This paper must be cited as:

Tavares De Araujo Cesariny Calafate, CM.; Fortino, G.; Fritsch, S.; Monteiro, J.; Cano Escribá, JC.; Manzoni, P. (2012). An efficient and robust content delivery solution for IEEE 802.11p vehicular environments. Journal of Network and Computer Applications. 35(2):753-762. doi:10.1016/j.jnca.2011.11.008.

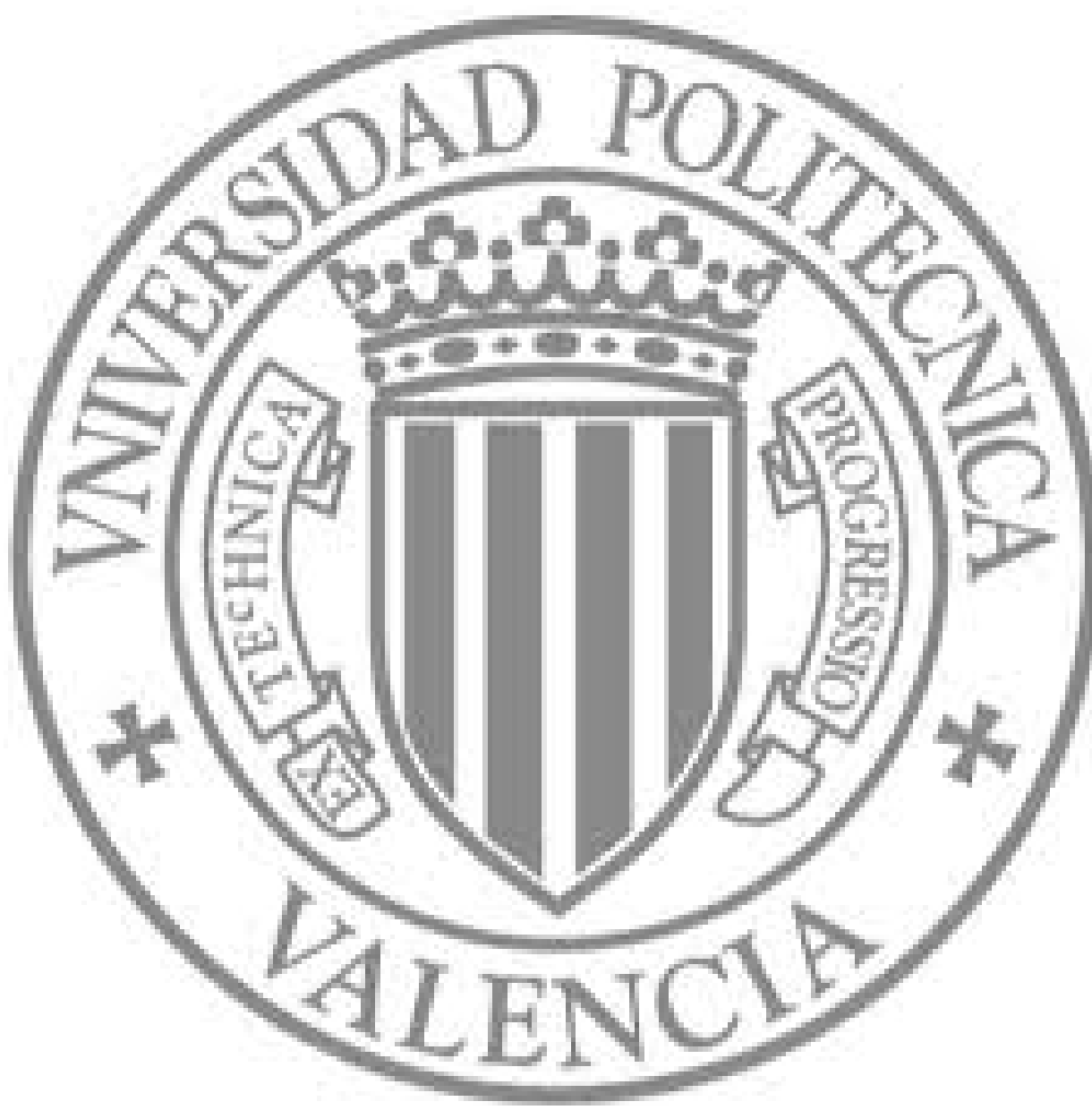

The final publication is available at

hppt://dx.doi.org/10.1016/j.jnca.2011.11.008

Copyright Elsevier 


\title{
An efficient and robust content delivery solution for IEEE 802.11p vehicular environments
}

\author{
Carlos T. Calafate ${ }^{\mathrm{a}, *}$, Giancarlo Fortino ${ }^{\mathrm{b}}$, Sascha Fritsch $^{\mathrm{c}}$, Janio Monteiro ${ }^{\mathrm{d}}$, Juan-Carlos Cano ${ }^{\mathrm{a}}$, \\ Pietro Manzoni ${ }^{\mathrm{a}}$ \\ ${ }^{a}$ Department of Computer Engineering, Universitat Politècnica de València, Spain \\ ${ }^{b}$ Dipartimento di Elettronica Informatica e Sistemistica, Università della Calabria, Italy \\ ${ }^{c} H f T$ Leipzig, Germany \\ ${ }^{d}$ Department of Electrical Engineering, University of Algarve, Portugal
}

\begin{abstract}
Vehicular networking is a new field that is expected to be widely adopted in the near future. One of the key applications inherent to this novel communications paradigm is content delivery to on-board users. In this paper we focus specifically on the design of a robust and efficient broadcastbased content delivery system. In order to reduce the content delivery time to a minimum, we first optimize performance by seeking the optimal packet size for content delivery. This goal is achieved by combining analytical and simulation results, and considering both static and mobile receivers at different distances from the transmitter. Moreover, we develop a full architecture that integrates the FLUTE protocol with different Forward Error Correction (FEC) schemes to achieve efficient content distribution. Through real experiments in a vehicular testbed we demonstrate that Raptor codes are the best option among the different FEC schemes available. In addition, as vehicle speed and/or distance from the broadcasting antenna increases, performance results highlight that adopting efficient FEC schemes becomes mandatory to achieve efficient and reliable data content delivery.
\end{abstract}

Keywords: IEEE 802.11p; vehicular networks; optimal packet size; content delivery framework; FEC techniques.

\section{Introduction}

Vehicular Area Networks (VANs) are currently a hot research topic that is receiving much attention from both industry and academia. Several projects focus on improving on-road safety by exploiting the vehicular networks concept using either vehicle-to-infrastructure (V2I) communications $[1,2]$, vehicle-to-vehicle (V2V) communications $[3,4,5]$ or the combination of both $[6,7]$.

In terms of technologies, a single standard has been developed specifically targeting on vehicular network environments, and supporting both V2I and V2V communication modes. This standard, known as IEEE 802.11p [8], is an enhancement to the original IEEE 802.11 standard that basically combines the IEEE 802.11a (operation in the $5 \mathrm{GHz}$ band) and the IEEE 802.11e (MAC-level QoS 
support) annexes [9]. In particular, the band of frequencies reserved for the operation of IEEE 802.11p is defined at $5.9 \mathrm{GHz}$, although there is still no worldwide consensus on the use of this band.

Besides supporting safety/emergency services in vehicular networks, the IEEE 802.11p standard also supports other types of services such as content delivery. In fact, content delivery in VANs is also expected to become a challenging research topic in the years to come.

When focusing on content delivery, we must differentiate between the unicast and broadcast cases. While in the unicast case we aim at giving service to the demands of a particular vehicle, usually through bidirectional communication, in the broadcast mode we aim at unidirectional delivery of contents to some set of users. Additionally, when relying on broadcast services, we can further differentiate between the most common systems, which include TV, radio, and webcasting, where information flow is continuous, and least common ones, where information is cyclically repeated to achieve reliable data delivery; examples of the latter include teletext systems, set-top box updates, and massive application delivery.

In this work, our focus is on reliable data delivery through broadcasting; in particular, we will optimize the delivery of advertisements to vehicles, being advertisements in the form of a file (e.g. picture, video) or a set of files (e.g. HTML page). To accomplish this goal we must address the specificities of vehicular environments. For instance, when cars are moving in urban environments, they have variable speeds, and also signal propagation is constrained by the configuration of the city structure [10]. Thus, if a road side unit (RSU) wants to propagate a block of information to a vehicle passing by, the most basic approach would be to periodically rebroadcast the same content. This approach has fundamentally two limitations, namely data synchronization and car speed. The first issue stands in the fact that, if the car starts receiving the block of information when the content distribution process has already began, it will have to wait for the beginning of the transmission loop to get the whole information. The second issue refers to the fact that, depending on the car speed and on the configuration of the city layout, the car will only be reached by the information sent by the specific RSU during a limited interval of time. In this setting, optimizing the content delivery process is of utmost importance to reach the greatest number of potential receivers before they get outside the delivery range. To meet this goal, the system manager can tweak the system, introducing two basic optimizations: determine the best packet size adopted for delivery, and determine the best Forward Error Correction (FEC) scheme to accelerate delivery in the presence of errors. In this work we focus on these optimizations in the scope of content distribution to moving vehicles using IEEE 802.11p. Our first contributions is a methodology to determine the optimal packet size for content delivery so as to achieve the maximum throughput possible when pushing broadcasted contents towards static or mobile receivers. Our second contribution is a comparison of the effectiveness of different FEC strategies in a real vehicular network testbed, using a fully operational application prototype. With this purpose we adopt different Forward Error Correction (FEC) techniques, and assess their effectiveness when varying the sender-receiver distance (static receiver) and the receiver speed (mobile receiver). Experimental results show that even the most simple FEC scheme is able to significantly improve performance compared to not using any FEC scheme at all.

The paper is organized as follows: in the next section we refer to some related works concerning content delivery in vehicular environments, packet size optimization and FEC techniques. In section 3 we describe our broadcast-based robust content delivery system, including an overview of our target vehicular network environment. In section 4 we combine an analytical framework with simulation results to determine the optimal packet size for transmission towards both static and 
mobile receivers. Experimental results obtained in a testbed are then presented and discussed in section 5. Finally, section 6 concludes the paper.

\section{Related works}

In this section we provide an overview of related works in the three research areas that we combine in this work: (i) content delivery solutions for vehicular environments; (ii) packet size optimization schemes; and (iii) Forward Error Correction techniques.

\subsection{Content delivery in vehicular environments}

In the literature we can find some relevant works that focus on content delivery to moving vehicles.

Infoshare [11] addresses the problem of information caching and delivery in intervehicular networks. It is based on a pull paradigm and application-level routing, and its goal is to achieve maximum spreading of information queries among vehicles. To achieve this goal it uses a smart caching policy that limits the overhead of useless queries and duplicated replies. The proposal is evaluated in a unidimensional network scenario (one-way road) using the ns-2 simulation tool.

Cabernet [12] is a solution for improving data delivery to moving vehicles that combines a streamlined client-side process, which establishes end-to-end connectivity in a very short time (less than $400 \mathrm{~ms}$ ), with the Cabernet transport protocol (CTP), which distinguishes congestion on the wired portion of the path from losses over the wireless link, two times throughput improvement over TCP can be achieved.

Huang et al. [13] propose a service-oriented information dissemination scheme to support vehicle infotainment that accounts for heterogeneous vehicular communication. They also develop a framework for delivering real-time services over an IP-based network to assure interoperability, roaming, and end-to-end session management. The effectiveness of their solution is shown using a self-developed simulation tool.

FleaNet [14] is a virtual marketplace where users can carry out buy/sell transactions on a vehicular network. In this solution sellers will disseminate queries, and resolutions are carried out through the FleaNet protocol suite, which is scalable to thousands of nodes. Authors evaluate their solution through both mathematical analyses and simulations, showing that random queries can be resolved within a tolerable amount of time.

Our solution differs from the existing ones by placing a strong emphasis on content locality, and by seeking to optimize unidirectional content delivery though packet size optimization and the adoption of FEC schemes at the application layer.

\subsection{Packet size optimization}

In the literature we can find works that focus on packet size optimization with different goals. In [15] authors study the optimal packet size for data communication in wireless sensor networks choosing energy efficiency as the optimization metric. The use of fixed size packets is proposed in light of the limited resources and management costs in sensor networks. In [16] authors study the effect of packet size on loss rate and delay characteristics in a wireless real-time application. Through both an analytical model and real experiments in an ad hoc network, they show that careful design of packetization schemes may significantly improve radio link resource utilization in delay sensitive media streaming under poor channel conditions. Other studies [17, 18] focus on the relationship between packet size and throughput, showing that adaptive sizing of the MAC 
layer frame in the presence of varying channel noise indeed has a large impact on the user seen throughput (goodput).

Packet size optimization gains special relevance in the context of vehicular networks since the high degrees of mobility inherent to these networks cause, on one hand, that the time during which radio coverage is available becomes quite limited and, on the other hand, that the radio channel conditions experienced by moving vehicles varies drastically throughout time. Thus, our work differs from the former ones by seeking packet size optimality for $802.11 \mathrm{p}$ vehicular settings, thereby addressing the challenges that make vehicular environments unique.

\subsection{Forward Error Correction techniques}

Forward Error Correction (FEC) is a transmission control system where the transmitter adds redundant data, called Error Correction Code [19], to identify and possibly correct an error in the transmission without requiring the transmitter's intervention. XOR is one of the simplest FEC schemes for error handling, being designed to ensure protection against the loss of a single packet in the presence of low error rates [20]. Its aim is to partition each Source block using a fixed Source Symbol length, and then adding redundant symbols built as the XOR sum of all Source Symbols. This process is called Encoding $(\mathrm{k}+1, \mathrm{k})$, where $\mathrm{k}$ is the number of Source Symbols. Since the XOR operation has little complexity, the time overhead introduced by this simple FEC process is minimal.

Reed-Solomon (R-S) [21] is a much more sophisticated FEC scheme that assumes an RS(N, K) code, which results in $\mathrm{N}$ codewords of length $\mathrm{N}$ symbols, each one storing $\mathrm{K}$ symbols of data, that are then sent over an erasure channel. Any combination of $\mathrm{K}$ codewords received at the other end is enough to reconstruct all of the $\mathrm{N}$ codewords. $\mathrm{N}$ is usually $2 \mathrm{~K}$, meaning that at least half of all the codewords sent must be received in order to reconstruct all of the codewords sent. Reed-Solomon is used in a series of commercial applications, including CDs and DVDs (to ensure their reading even with minor scratches), and also in broadcasting satellite systems, DSL, WiMax, and Digital Terrestrial TV (DVB-T). Concerning the time overhead introduced, R-S FEC creates codeworks through polynomial multiplication techniques, which introduces quite more overhead than the XOR technique. However, there is a lot of hardware available that allows accelerating R-S FEC encoding and decoding processes; thus, the overhead involved is moderate and can not be considered as problematic.

More recently, Raptor encoding has emerged as the most efficient FEC technique currently available. The Raptor acronym stands for Rapid Tornado, and represents an evolution of the first types of "Erasure Codes". The technique was invented by Amin Shokrollahi in 2001 [22], and is based on the concept of Luby Transform, which uses a parity array, being considered the first efficient method for encoding a block of $\mathrm{n}$ symbols in $\mathrm{O}(\mathrm{n})$ time. Raptor has a high efficiency because it uses multiple levels of encoding (and decoding): in the first level it performs a "pre-coding" on Source Blocks, thus generating intermediate symbols; although different pre-coding algorithms may be used, the most widely adopted is generally the LDPC (Low Density Parity Check). Afterwards, intermediate symbols are processed using LT-Codes (Luby Transform) to generate the final encoded symbols (Repair Symbols). A big advantage of Raptor codes is that, with the Luby Transform, an unlimited number of Repair Symbols can be generated, offering a great flexibility to transmission systems. In terms if time overhead, though, the relatively high complexity of the process, associated with the need to perform encoding in two steps, introduces a relatively high delay, even when using powerful processors. In the scope of our work, though, contents need to be encoded only once since the Raptor technique allows generating a potentially unlimited number of recovery symbols. Thus, 


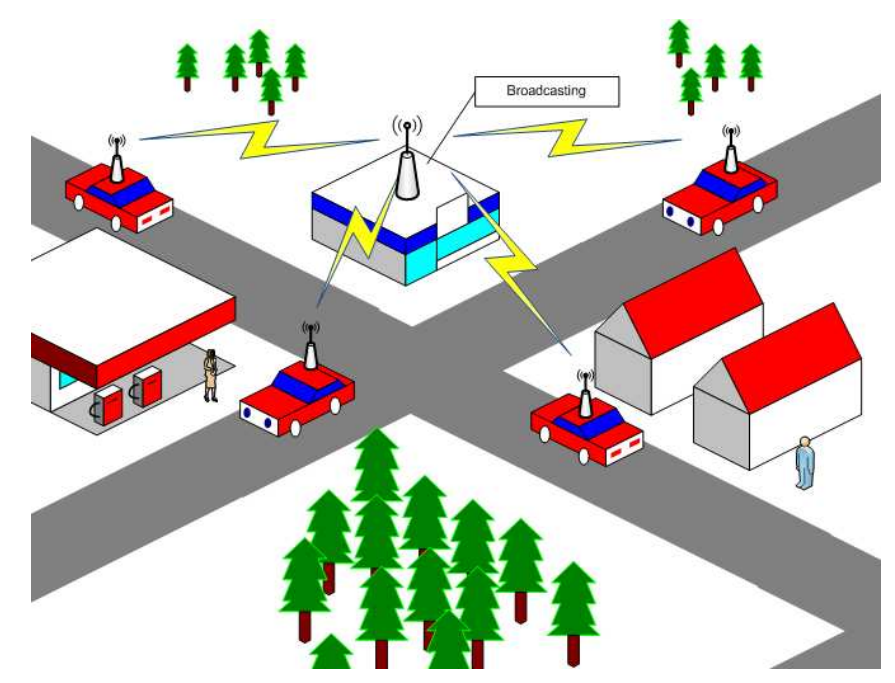

Figure 1: Illustration of the target scenario: a small business broadcasts announcements to passing-by vehicles within a strictly limited range.

the time overhead introduced is mainly associated with the startup process, and does not hinder performance.

\section{A BROADCAST-BASED ROBUST CONTENT DELIVERY SYSTEM}

In this section we describe the proposed content delivery system. With this aim, we first describe the target environment for our application; afterwards, we proceed to provide a detailed description of the delivery system itself.

\subsection{Description of the target environment}

The envisioned target environment for this work focuses on a specific Vehicular Area Network scenario where corporate business servers push contents such as advertisement information (e.g. daily offers) to passing-by vehicles interested in that sort of information. With this purpose these servers employ RoadSide Units (RSUs) endowed with an omnidirectional antenna, which is used for broadcasting data using IEEE 802.11p technology (see figure 1).

The envisioned system falls under the Vehicle-to-Infrastructure (V2I) communications category, although the infrastructure is limited to a single antenna with very limited reachability. Our scenario includes (i) urban environments, where buildings are more packed together, vehicle density is higher and vehicle speed is low, and (ii) roads populated with roadside commercial areas, where buildings are relatively far away from each other, vehicle density is lower and vehicle speed is higher.

Drivers interested in receiving advertisements of a certain category (hotels, gas stations, restaurants, etc.) should configure their on-board units (OBUs) accordingly. When received completely, the data is presented to the driver on the OBU's display.

Concerning the information to be pushed into vehicles, it shall consist of a multimedia file (video, html page, picture with text, etc.). 


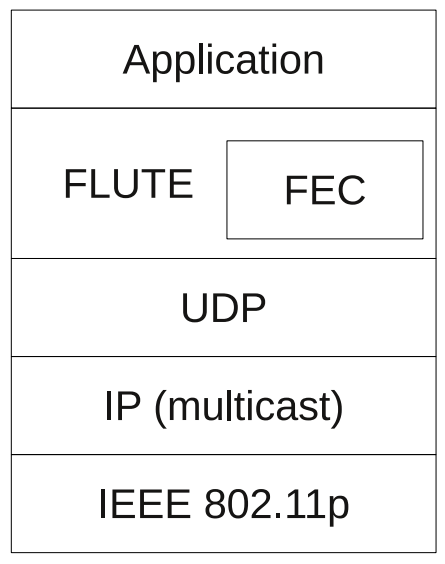

Figure 2: System Architecture.

\subsection{Details about the delivery system}

The developed system combines the functionality of the FLUTE protocol with the features of FEC schemes to provide a robust and reliable content delivery system for wireless urban environments; such system is also able to reduce file transfer times.

FLUTE (File Delivery over Unidirectional Transport) [23] is a protocol defined for broadcasting (or multicasting) data. It does not require bidirectional communication between sender and receiver, thus working with any network supporting unidirectional communications (Internet, satellite, WiFi, etc). In particular, it builds upon the ALC (Asynchronous Layered Coding) standard [24], thus allowing to obtain great scalability in multicast-based environments by performing a segmented delivery of files to many hosts simultaneously. Since the information will be broadcasted, the system can transparently scale to any number of receivers. Since the receivers come within transmission range at unpredictable times, the delivered contents are transmitted over and over again in a loop, which is only broken if contents are deleted or updated.

The layered architecture of the proposed system is shown in figure 2. At the application layer we have developed two different components: the broadcaster, located at the static base station side, and the receiver, located at the mobile client side. Both applications are based on the FLUTE protocol, and may rely on different FEC schemes (XOR, Reed-Solomon, or Raptor) to make data transfer faster, more robust, and more reliable.

Concerning the MAC/PHY layers, we rely on 802.11p technology, although the $802.11 \mathrm{a}$ technology could be used as well since it adopts the same band and has similar transmission properties, while offering a greater number of available channels. Notice that, since the IEEE 802.11p standard defines channels for both critical and non-critical traffic, this sort of applications can fit well within the scope of the standard. With regard to actual deployment, a dedicated channel where channel contention is not expected would be preferable. That would mean that losses would depend mostly on the characteristics of the channel as experienced by each vehicle. If the channel is shared by different RSUs within each other's coverage range, he CSMA/CA medium access algorithm promotes the statistical sharing of bandwidth among the different RSUs, does not representing a problem. If, however, RSUs are not within range of each other, causing partial coverage area overlapping, then channel sharing is not advisable since collisions are more prone to occur; in those situations, channel allocation schemes should be adopted [25]. Additionally, since the optimization process 


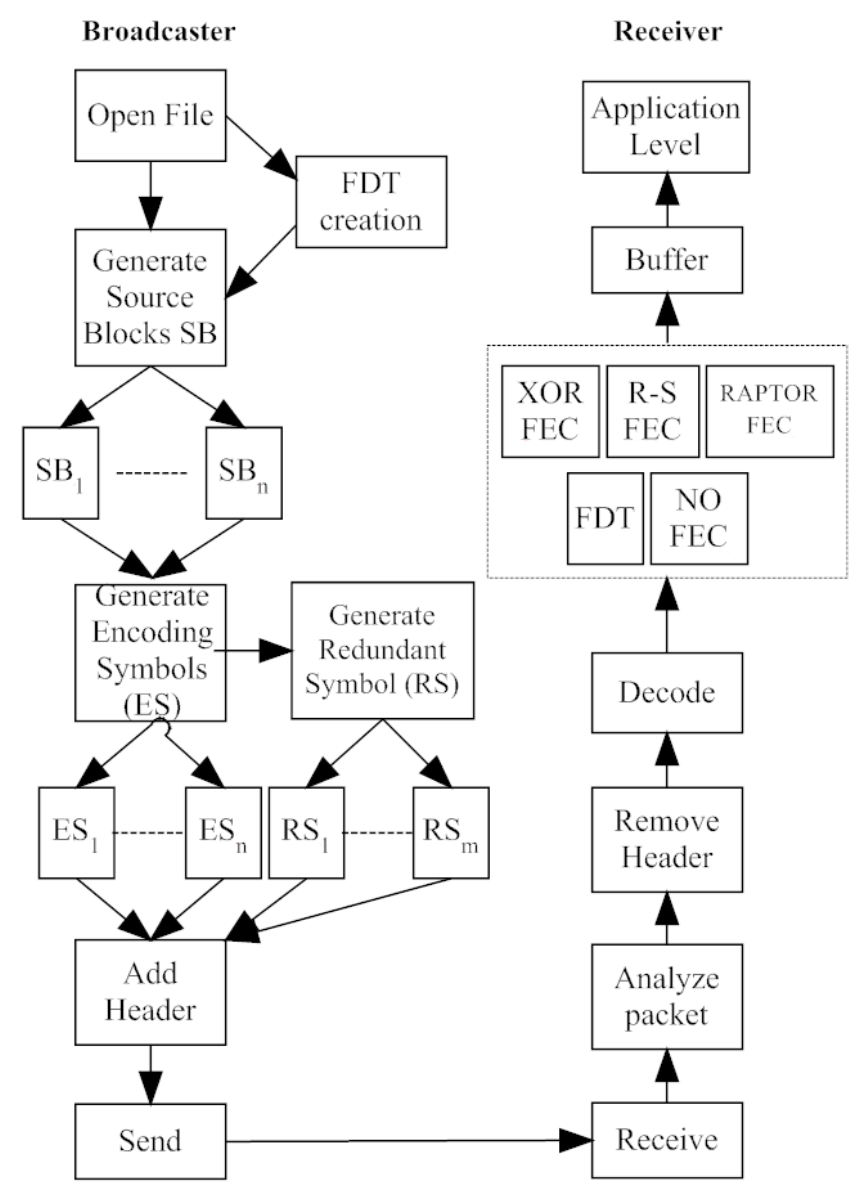

Figure 3: Schema of the broadcaster/receiver activity flow.

becomes context and receiver specific, in situ measurements of the packet loss ratio at different positions along the target street or road is recommended, as referred in section 4 .

It is also important to have in mind that, contrarily to unicast transmissions, where data rate adjustments or even packet size adjustments allow adapting to the fluctuating nature of the wireless channel on a per-receiver basis, the broadcast nature of our solution prevents such approaches, thus requiring a more general solution to achieve optimal performance.

In our solution, the broadcaster performs the following operations (Figure 3):

1. Opening the file to-be-transmitted in binary mode.

2. Data characterization and creation of the FDT (File Delivery Table). FDT instances, together with FLUTE header fields, give the necessary parameters to identify, locate, and restore the files at the receiver.

3. Decomposition into source blocks. In particular, the decomposition process adopted by the FLUTE algorithm works as follows: computation of a source block structure so that all source blocks are as close to being of equal length as possible; all source blocks in the first group 
share a same larger length, while the second source blocks group is associated with a smaller length.

4. Decomposition in encoding symbols (only source symbols).

5. Addition of redundancy through FEC, if enabled. The integrated FEC schemes are XOR, R-S and Raptor (see section 2). Thus, the encoded symbols will be the source symbols plus the redundancy symbols.

6. Construction and transmission of the FLUTE packets.

The receiver carries out the inverse operations of the broadcaster (Figure 3):

1. Waiting for the FDT to be received.

2. Parsing the FDT and saving the related parameters.

3. Storing incoming data packets.

4. Analysis of the received packets.

5. Data decoding: in case of redundancy, it is added by the sender and saved into a buffer.

6. Handing over the file to the application level as soon as it is fully received.

The system is fully implemented in C under Linux (Debian) and it extends the FLUTE library mad-fcl 1.7 [26], which already provides Compact No-Code, XOR, and R-S FEC schemes, to achieve integration with the Raptor library [27].

\section{Tuning the packet size for optimal delivery effectiveness}

Taking the target environment described in section 3 as reference, we now proceed to determine which packet size allows optimizing the effectiveness of the content delivery process at any given sender-receiver distance when relying on the IEEE $802.11 \mathrm{p}$ technology. Notice that different packet size choices will have an impact on both the achievable throughput and the packet loss, especially when considering that large packet sizes have little chances of being successfully delivered over long distances.

In urban areas, there are several obstacles and reflectors on the radio channel that cause fading to occur frequently. Thus, it often occurs that there is no line of sight between transmitter and receiver. In case line of sight exists, multi-path effects will still take place due to reflection from the ground and surrounding objects.

The multipath components combine vectorially at the receiver antenna, causing the received signal to distort or fade. Due to the constructive and destructive effects of multipath waves, a receiver moving at high speed can go through several levels of fading in a small period of time.

We surveyed the literature to determine which would be the most appropriate radio propagation model for that environment. Considering the results presented by Killat and Hartenstein [28], the Nakagami distribution matches empirical data better than other models. Thus, we picked the Nakagami fading model with $m=3$ for our experiments. Notice parameter $\mathrm{m}$ is called the 'shape factor' of the Nakagami-m distribution, controlling the severity, or depth, of the amplitude fading.

The two following subsections will focus on packet size optimality for static and dynamic receiving vehicles, respectively. 


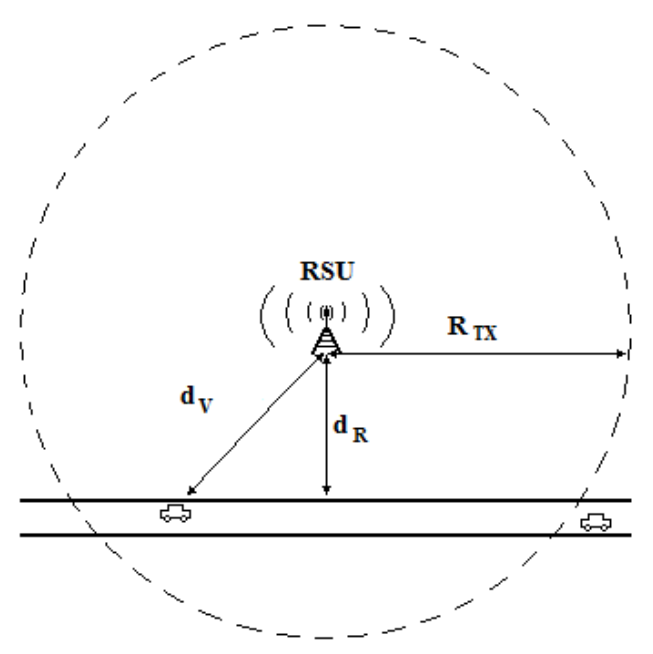

Figure 4: Illustration of the distances associated with the analytical study.

\subsection{Analysis for a static receiver}

Consider that $\phi(L)$ is a function that describes the maximum layer-three load that can be injected into the wireless medium using IEEE $802.11 \mathrm{p}$ for a datagram length of $L$ bytes when assuming broadcast mode and a raw data rate of $6 \mathrm{Mbit} / \mathrm{s}$ (default value when broadcasting on a $20 \mathrm{MHz}$ channel). Additionally, consider that $\psi(L, d)$ describes the packet reception probability as a function of distance $(d)$ and packet size $(L)$ according to the Nakagami-3 fading model, which is considered to adequately grasp the channel propagation behaviour in vehicular environments [28]. Our approach can also be adopt real in situ measurements as an alternative to using the Nakagami fading model in those situations where several sources of interference are present in the target scenario.

The main goal is to find, for any Vehicle-to-RSU distance $\left(d_{V}\right)$ - see figure 4 -, which packet size is able to maximize the throughput at the receiver. In analytical terms, we seek a packet size $L_{o}$ that satisfies the following condition:

$$
\begin{gathered}
\phi\left(L_{o}\right) \cdot \psi\left(L_{o}, d_{V}\right) \geq \phi\left(L_{i}\right) \cdot \psi\left(L_{i}, d_{V}\right), \\
\forall L_{o}, L_{i} \in \mathbb{N}: L_{\min } \leq L_{i}, L_{o} \leq L_{\max }, L_{o} \neq L_{i}
\end{gathered}
$$

being that $L_{i}$ refers to all other packet sizes that differ from the optimal one. Notice that, for the frames based on the IEEE 802.11 technology, the minimum and maximum datagram sizes that can be encapsulated by the MAC layer frame are $L_{\min }=64$ bytes and $L_{\max }=2308$ bytes.

To achieve our goal we must first derive functions $\phi(L)$ and $\psi(L, d)$. Focusing first on the relationship between datagram length and throughput - $\phi(L)$ - we first obtained several reliable samples using the ns-2 simulator [29], which were then used to determine the maximum achievable throughput for each packet size (see Figure 5). Notice that these values are associated with a broadcast transmission, meaning that they are independent of both the number of receivers and the distance towards those receivers. Also, since there is no type of feedback returned by the receiver, broadcasted transmissions do not employ RTS/CTS exchanges at the beginning of the 


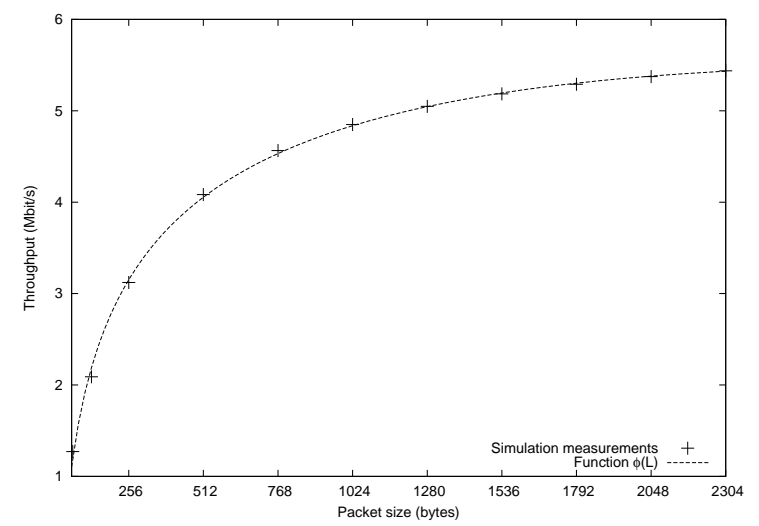

Figure 5: Maximum achievable throughput for each packet size when broadcasting using IEEE 802.11p technology (6 Mbit/s raw data rate, $20 \mathrm{MHz}$ channel, lossless transmission).

transmission, nor of Acknowledgment packets at the end. Thus, the transmission model is greatly simplified compared to unicast scenarios.

The throughput values shown in Figure 5 range from $1.27 \mathrm{Mbit} / \mathrm{s}$ for a frame carrying a datagram of 64 bytes, and up to $5.44 \mathrm{Mbit} / \mathrm{s}$ for a frame carrying a datagram of 2304 bytes.

Throughout our analysis we have tested different families of functions to determine one that was both simple and accurate. Finally, we opted for the following function:

$$
\phi(L)=\alpha \cdot \log (\beta \cdot L)+\delta \cdot L+\epsilon
$$

where $(\alpha, \beta, \delta, \epsilon)$ are regression variables. This function allowed us to obtain a highly accurate regression curve (see Figure 5).

Concerning function $\psi(L, d)$, we could not find it in the literature since most radio propagation models focus on the relationship between signal-to-noise ratio (SNR) or Signal to Interference plus Noise Ratio (SINR) and Bit Error Ratio (BER). For the specific case of Nakagami-3 propagation, the model included in ns-2 does relate packet error probabilities with distance for the specific case of vehicular environments based on IEEE $802.11 \mathrm{p}$ technology. However, the loss model does not take packet size into account, thus being insufficient to achieve our goals. Instead, we adopted an approach that required taking the three following steps:

I) Obtain the Nakagami-3 fading model according to a distance-loss behaviour for a known packet size.

II) Obtain a function that represents the fading model behaviour in step I through regression.

III) Derive an analytical model that allows mapping the loss model for one packet size to any alternative packet size.

Concerning Step I, Killat and Hartenstein [28] provided us with the distance-success ratio behaviour for the Nakagami-3 fading model for a packet size of 382 bytes. We have tuned ns- 2 to be able to reproduce a similar behaviour (see table 1). This way we were able to overcome some of the limitations of the simulator's model in terms of accuracy, thus achieving a higher resemblance with real-life scenarios.

Based on ns-2 experimental measurements, we proceeded to Step II where we did tests with different families of functions to determine the most adequate one. In our case, we found that the 
Table 1: ns-2 simulation parameters.

\begin{tabular}{|c|c|}
\hline Parameter & Value \\
\hline \hline Frequency & $5.9 \mathrm{GHz}$ \\
\hline Tx Power & $20 \mathrm{dBm}$ \\
\hline Tx range & $400 \mathrm{~m}$ \\
\hline Noise floor & $-96 \mathrm{dBm}$ \\
\hline CS Threshold & $-94 \mathrm{dBm}$ \\
\hline SINR Preamble capture & $5 \mathrm{~dB}$ \\
\hline SINR Data capture & $10 \mathrm{~dB}$ \\
\hline
\end{tabular}

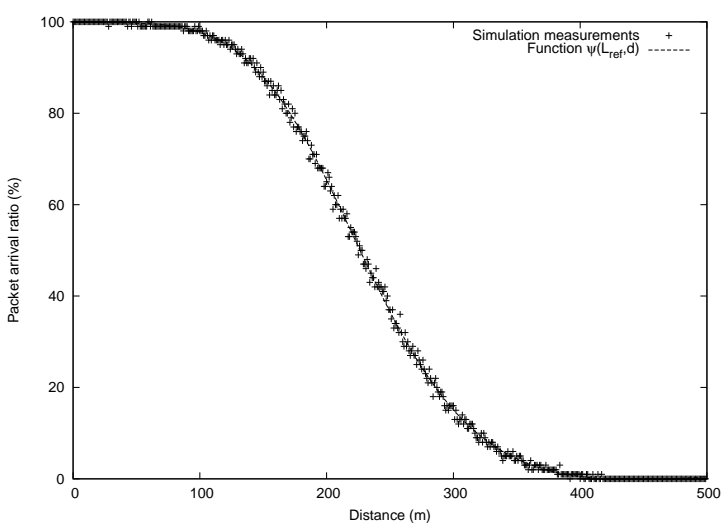

Figure 6: Packet arrival ratio at different source-destination distances for the Nakagami-3 fading model (packet size of 382 bytes).

following family of functions offer a very good trade-off between efficiency and accuracy:

$$
\psi\left(L_{r e f}, d\right)=\frac{\zeta \cdot d^{3}+\eta \cdot d^{2}+\theta \cdot d+\vartheta}{\kappa \cdot d^{3}+\lambda \cdot d^{2}+\mu \cdot d+\nu}
$$

The value of variables $(\zeta, \eta, \theta, \vartheta, \kappa, \lambda, \mu, \nu)$ was obtained through regression, and $L_{r e f}=384$ bytes. Figure 6 shows the samples along with the curve obtained through regression. The high fit accuracy obtained evidences the adequacy of the chosen family of functions. In general, each different combination of technology and deployment environment will require a tuning of the regression variables to accurately grasp the behaviour of the channel.

Finally, in Step III, we developed an analytical model that relates the packet loss ratio for a certain packet size with that for any other size. Our point of departure to achieve this goal was the equation presented in $[18,30]$ :

$$
P_{e}^{m}\left(l, \gamma_{s}\right)=1-\left(1-P_{u}^{m}\left(\gamma_{s}\right)\right)^{l}
$$

which relates $P_{e}^{m}\left(l, \gamma_{s}\right)$, the packet error rate for a packet size $l$, with $P_{u}^{m}\left(\gamma_{s}\right)^{l}$ (the bit error rate), where $\gamma_{s}$ refers to the SNR value per symbol and $m$ is the Nakagami PHY mode. Notice that $l$ represents the packet size in bits, thus differentiating it from $L$, which we use to represent packet size in bytes instead. 


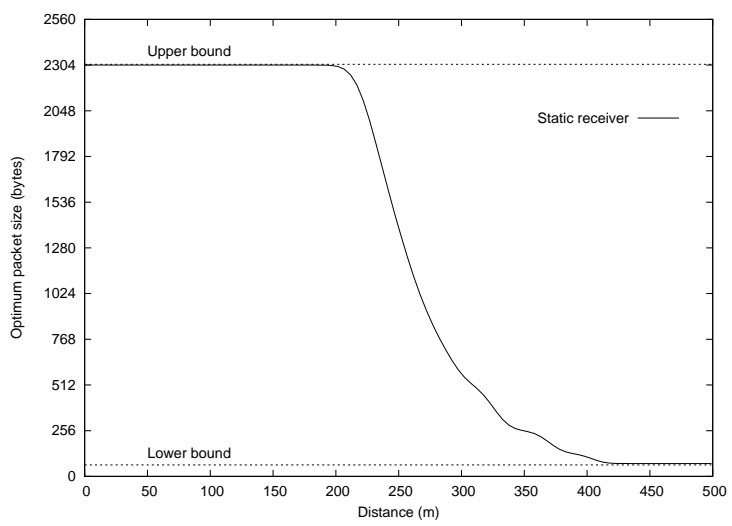

Figure 7: Optimum packet size for a static vehicle at different distances considering the Nakagami-3 fading model.

Since this expression specifically addresses Nakagami radio links, in Appendix A we derive the packet size mapping formula that allows relating packet error probabilities for different packet sizes. The expression achieved for the packet error ratio is the following:

$$
P_{e}^{m}\left(l_{2}, \gamma_{s}\right)=1-\left[1-P_{e}^{m}\left(l_{1}, \gamma_{s}\right)\right]^{\frac{l_{2}}{l_{1}}}
$$

where $l_{1}$ and $l_{2}$ are two different packet sizes. For the packet arrival ratio we have instead:

$$
P_{s}^{m}\left(l_{2}, \gamma_{s}\right)=\left[P_{s}^{m}\left(l_{1}, \gamma_{s}\right)\right]^{\frac{l_{2}}{l_{1}}}
$$

where $P_{s}^{m}\left(l_{2}, \gamma_{s}\right)$ and $P_{s}^{m}\left(l_{1}, \gamma_{s}\right)$ refer to the packet arrival probabilities for packet lengths $l_{2}$ and $l_{1}$, respectively. By replacing $P_{s}^{m}\left(l_{1}, \gamma_{s}\right)$ by $\psi\left(L_{r e f}, d\right)$ in the former expression, we can finally derive the generic expression for $\psi(L, d)$ :

$$
\psi(L, d)=\left(\frac{\zeta \cdot d^{3}+\eta \cdot d^{2}+\theta \cdot d+\vartheta}{\kappa \cdot d^{3}+\lambda \cdot d^{2}+\mu \cdot d+\nu}\right)^{\frac{8 \cdot L}{l_{r e f}}}
$$

where $l_{\text {ref }}$ refers to our reference packet size (382 bytes) expressed in bits.

Finally, replacing equations 2 and 7 in expression 1, we are now able to find the optimal packet size $\left(L_{o}\right)$ for any Vehicle-to-RSU distance $\left(d_{V}\right)$. The values achieved are presented in figure 7 (static receiver). We can see that, for distances up to 200 meters, throughput can be maximized by picking the maximum packet size available. However, beyond that distance, this rule no longer applies, being that the optimal packet size decreases with increasing distance. In particular, the lower bound is achieved for a distance of about 420 meters. Finally, we should mention that, in a real scenario, the transmitting terminal is unaware of the distance towards the receivers since all communications are broadcast-based (similarly to, e.g., digital television). Thus, we seek a global optimization that is applicable to all possible receivers independently of their position. In particular, we will base our optimization on the distance between the RSU and the road itself (parameter $d_{R}$ in Figure 4). This topic is addressed in the following section. 


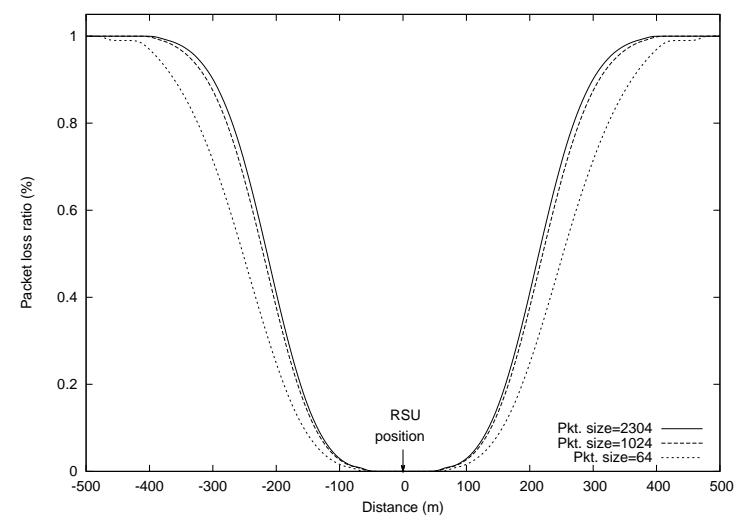

Figure 8: Packet loss ratio at different distances with different packet sizes (assuming a Road-to-RSU distance of 5 meters).

\subsection{Analysis for a mobile receiver}

Based on the analysis presented in the previous section, we now extend the model to the more typical and more challenging situation where the receiver (in our case a vehicle) is moving. The results obtained in this setting allow determining the optimal packet size setting for any given distance between the RSU and the road itself, regardless of the actual speed or the number of vehicles.

Figure 8 shows the channel conditions experienced by the receiver as the distance from the RSU and the packet size change. We can see that a moving vehicle initially experiences a high packet loss ratio, but then the packet loss ratio falls sharply when approaching the RSU. The opposite trend is experienced as the vehicle moves away from the RSU.

As expected, smaller packet sizes experience a smaller packet loss ratio at all distances. However, since larger packets carry more data, it is unclear which is the optimal packet size for any given Road-to-RSU distance $\left(d_{R}\right)$ - see figure 4 .

We now introduce an analytical model that is able to grasp the network conditions experienced by a moving vehicle. From this model we are able to derive the optimum packet size for content delivery to moving vehicles for any given value of $d_{R}$. With this purpose we first derive an equation that relates $d_{R}$ with the Vehicle-to-RSU distance $\left(d_{V}\right)$. Since in our scenario the channel behaviour experienced by vehicles is symmetric, for the subsequent analysis is it enough to consider only half the trajectory. Thus, considering that the vehicle is at a distance $d_{R}$ from the RSU at $t=0$ :

$$
d_{V}\left(t, d_{R}\right)=\sqrt{(\nu \cdot t)^{2}+d_{R}^{2}}
$$

By applying this formula to equation 7 and integrating over time, we obtain:

$$
\chi\left(L, d_{R}\right)=\int_{t=0}^{\infty} \phi(L) \cdot \psi\left[L, d_{V}\left(t, d_{R}\right)\right] \partial t
$$

which allows to quantify the amount of data received while in the vicinity of the RSU. Thus, for a given Road-to-RSU distance $\left(d_{R}\right)$, the optimum packet size $L_{o}$ is such that : 


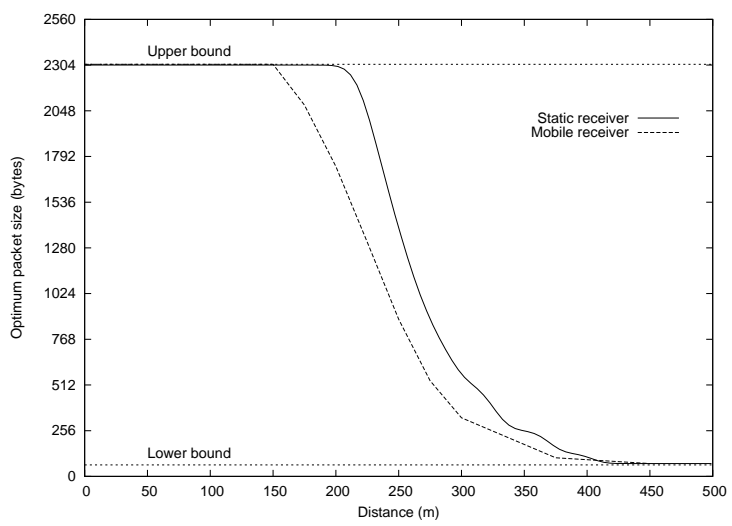

Figure 9: Optimum packet size at different distances for the Nakagami-3 fading model for static and mobile receivers.

$$
\begin{gathered}
\chi\left(L_{o}, d_{R}\right) \geq \chi\left(L_{i}, d_{R}\right), \\
\forall L_{o}, L_{i} \in \mathbb{N}: L_{\text {min }} \leq L_{i}, L_{o} \leq L_{\text {max }}, L_{o} \neq L_{i}
\end{gathered}
$$

Equation 10 now enables us to derive the optimum packet size $\left(L_{o}\right)$ at different Road-to-RSU distances regardless of the actual speed of vehicles. These values are shown in Figure 9.

For comparison purposes, Figure 9 also includes the results for a static receiver previously obtained. However, contrarily to the static receiver case, the distance represented in the $x$ axis is the Road-to-RSU distance $\left(d_{R}\right)$, and not to the distance between the vehicle and the RSU $\left(d_{V}\right)$, which now varies throughout time. From this figure we find that, for $d_{R}$ distances below 150 meters, the optimum packet size is the maximum one available (2308 bytes). Afterward it decreases, approaching the minimum value (64 bytes) for $d_{R}$ values close to 450 meters.

Notice that the actual Vehicle-to-RSU distance $\left(d_{V}\right)$ is always equal to or greater than the Roadto-RSU distance $\left(d_{R}\right)$, which explains why the optimum values for mobile receivers are lower than the ones for static receivers. Also, despite the fact that large packet sizes experience more losses for large distances, they are shown to be more effective since most of the information is received when the vehicle is closer to the RSU. Going back to figure 8, notice that most data are received when the vehicle is within a \pm 200 meters range of the RSU position.

Taking into account the optimal packet size under mobility conditions shown in figure 9, we now obtain an upper bound for the raw amount of data that a vehicle can receive when passing by the coverage region of an RSU broadcasting contents. These results are presented in figure 10; we can see that, as expected, both Road-to-RSU distances and vehicle speeds have a significant impact on the total amount of data received, being vehicular speed the most significant factor for small distances (less than 150 meters), and channel fading the most significant factor for large distances (more than 150 meters).

\section{Performance results in a real testbed}

We evaluated the proposed system with the aim of measuring the file transfer time under different static and mobile conditions of the receiver with respect to the broadcaster, and using 


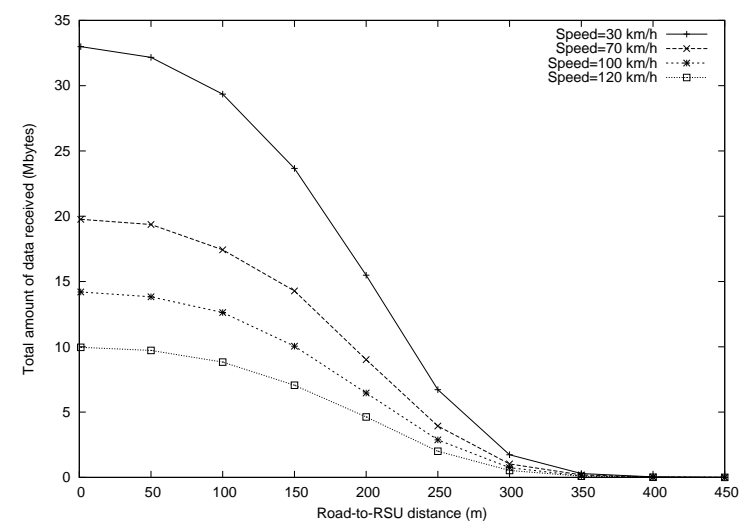

Figure 10: Total amount of data received for different Road-to-RSU distances at different vehicle speeds.

different FEC codes incorporated in the system. In particular, the experiments were run on two laptops communicating through direct WiFi $802.11 \mathrm{~g}$, one coupled with an omnidirectional antenna to provide a transmission range of approximately 500 meters and executing the broadcaster, and the other one mounted in a vehicle acting as the receiver. All tests were based on the following common parameters: Broadcast transmission rate $=1 \mathrm{Mbps}$, File to-be-transmitted $=254 \mathrm{~KB}$, and the the transmission process runs in an infinite loop.

We deployed our system in the campus of the Technical University of Valencia, locating the RSU near the street used for our tests (see figure 11). The broadcasting antenna was positioned at the top of a building, at $15 \mathrm{~m}$ height from the ground, and 20 meters away from the road. The packet size adopted for each experiment was picked according to the findings of section 4 to maximize throughput; in particular, we used the values shown in figure 7 as reference. Two kinds of experiments were set up: (i) static receiving vehicle; (ii) mobile receiving vehicle. In the first type of experiment, $\mathrm{t}$ whereas the receiver laptop was located at different distances from the broadcaster: $1 \mathrm{~m}, 100 \mathrm{~m}, 200 \mathrm{~m}, 300 \mathrm{~m}, 400 \mathrm{~m}, 500 \mathrm{~m}$. The first three distance values were set up with line-of-sight between broadcaster and receiver, while the last three distances had no line-of-sight.

The packet size chosen for each experiment varied according to the optimal packet size settings found analytically, and depicted in figure 7 . This means that, up to 200 meters, the maximum possible size was picked, while for the other three experiments the packet size was decreased.

The obtained results are reported in figure 12. In this test, the distance of $1 \mathrm{~m}$ was introduced just for reference since it is not a possible value in our context. The best results are provided by the NULL FEC, since it does not introduce any redundancy and computational complexity and losses are null. Between $100 \mathrm{~m}$ and $200 \mathrm{~m}$ there are minor performance difference among the 4 schemes, being that Raptor and R-S perform on average better than the NULL FEC and XOR FEC by about $23 \%$ and $13 \%$, respectively. Notice that, in this range, the packet loss ratio is still not very high, which explains why improvements are only slight. However, from $200 \mathrm{~m}$ to $500 \mathrm{~m}$, the use of FEC schemes leads to a considerable improvement. As can be seen, Raptor is always the best performing scheme. In particular, at $500 \mathrm{~m}$, Raptor outperforms the NULL FEC, XOR and R-S by about $180 \%, 69 \%, 11 \%$, respectively. This occurs because Raptor encoding is the most flexible and efficient FEC technique available, allowing almost any recovery packet to contribute to the original message reconstruction, a situation that does not apply to the other schemes. Moreover, 


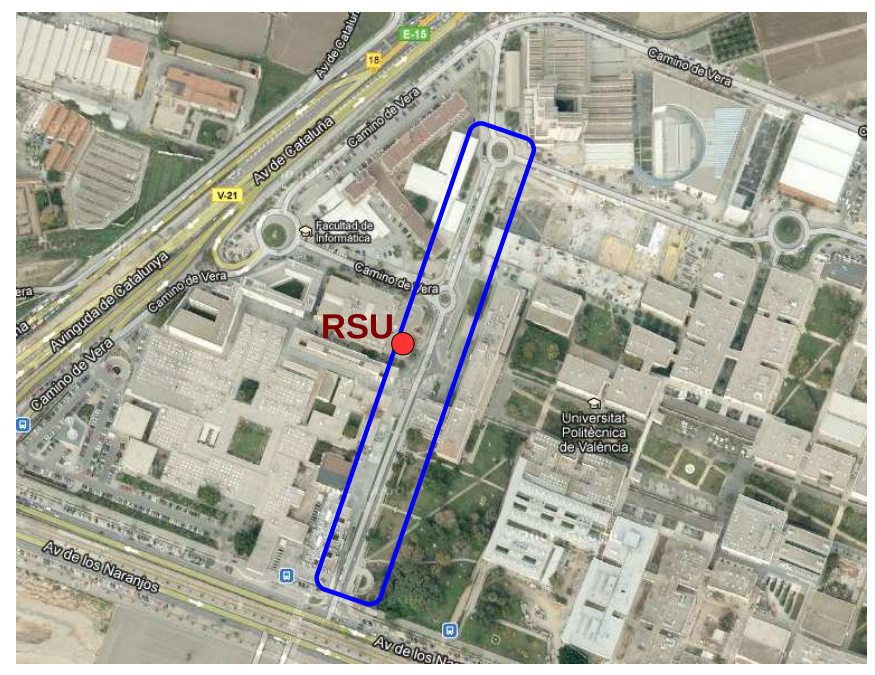

Figure 11: Target deployment area at the UPV campus (Valencia, Spain).

it is worth noting that, even when relying only on XOR (the simplest FEC scheme), we improve performance by about $66 \%$ with respect to NULL FEC. This suggests that, as a minimum, the XOR FEC scheme should be adopted to reduce file transfer time in these scenarios.

In the second type of experiment, the receiver laptop was located inside a car that circulated with different speed ranges: $10-20 \mathrm{Km} / \mathrm{h}, 20-40 \mathrm{Km} / \mathrm{h}, 40-60 \mathrm{Km} / \mathrm{h}, 60-80 \mathrm{Km} / \mathrm{h}$. The packet size chosen for these experiments was the maximum one available, according to figure 12 . Notice that, since the operating system imposes further MTU restrictions that impede using the maximum theoretical size of 2308 bytes defined for 802.11 interfaces, the actual MTU used matches the one for Ethernet, that is, 1500 bytes, which include both IP and UDP headers. The obtained results are reported in Fig. 13. They confirm the same trend as the ones obtained in the first experiment, being Raptor the FEC scheme providing the highest performance. In fact, the differences between Raptor and Reed-Solomon now become more evident, which is basically associated with the greater error resilience of the former at higher packet loss ratios. At a speed of $60-80 \mathrm{Km} / \mathrm{h}$ the file transfer time with Raptor FEC is of $11.4 \mathrm{~s}$ on average, so the mobile receiver can even travel straight for about $230 \mathrm{~m}$ at an average speed of $70 \mathrm{Km} / \mathrm{h}$ without stopping, being timely served by the broadcaster. It is worth noting that, when the speed range increases, the performance percentage gain of the XOR scheme with respect to the NULL FEC scheme is reduced. This implies that it may be unsuitable for urban environments in which mobile receivers can travel at a speed higher than $10-20 \mathrm{Km} / \mathrm{h}$, since the error resilience properties of this simple FEC mechanism are too weak when facing poor channel conditions.

\section{Conclusions}

Wireless content delivery infrastructures in urban environments are emerging to provide a wide range of contents (e.g. advertising, entertainment, traffic and parking notifications, and emergency announcements) to moving vehicles, as well as roaming people. In this paper we proposed a robust 


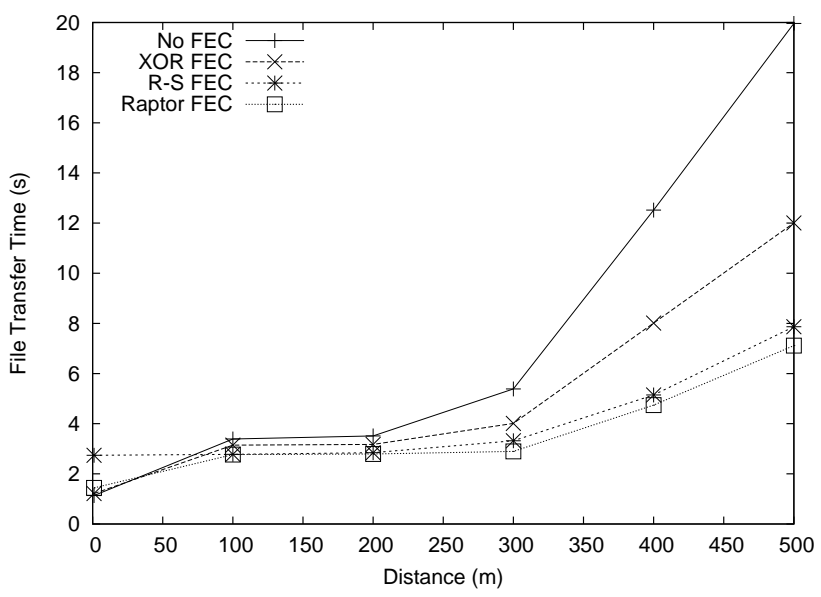

Figure 12: File transfer time for static receiving vehicles by varying distance and FEC scheme type.

content broadcasting system for the delivery of multimedia-based advertisement information (e.g. daily offers) to passing-by vehicles and people in urban environments.

By assuming a Nakagami fading channel to model packet loss at different distances using $802.11 \mathrm{p}$, we combined analytical and simulation results to determine the optimal packet size for transmission at different Road-to-RSU distances considering both static and mobile receivers.

Experimental results for static receivers show that throughput can be maximized by picking the maximum packet size available for distances up to 200 meters. However, beyond that distance, the optimal packet size decreases with increasing distance, achieving the lower bound for a distance of 420 meters. In the context of mobility, our optimization strategy has the advantage of allowing to determine an optimal packet size value that applies regardless of the actual speed of the different vehicles. Mobile receiver tests show that the optimum packet size for road-to-RSU distances up to 150 meters is the maximum one available, decreasing afterwards up to 450 meters. Differences towards the static receiver scenario are expected since, with mobile receivers, the actual vehicle-toRSU distance varies throughout time.

Based on our findings relative to packet size optimality under different conditions, we then proceed to deploy the proposed system in a real environment (testbed). The system integrates the FLUTE protocol for partitioning data into blocks, along with three FEC schemes (i.e. XOR, R-S, and Raptor) to make content distribution more robust, reliable and fast.

Performance evaluation tests were carried out in two different vehicular scenarios: one under static conditions, and another one under dynamic conditions. The obtained results show that the Raptor FEC scheme offers the best performance. Moreover, it is quite evident that the use of a FEC code, even the simplest one like the XOR FEC scheme, should be adopted as it allows improving performance by about $66 \%$ with respect to the non-exploitation of FEC schemes. Additionally we find that, if vehicles travel at speeds in the range $60-80 \mathrm{Km} / \mathrm{h}$ (typical or urban scenarios), the R-S and Raptor schemes represent the only viable choice.

In the future we plan to develop OBU-specific middleware to implement the transmission strategy here presented. 


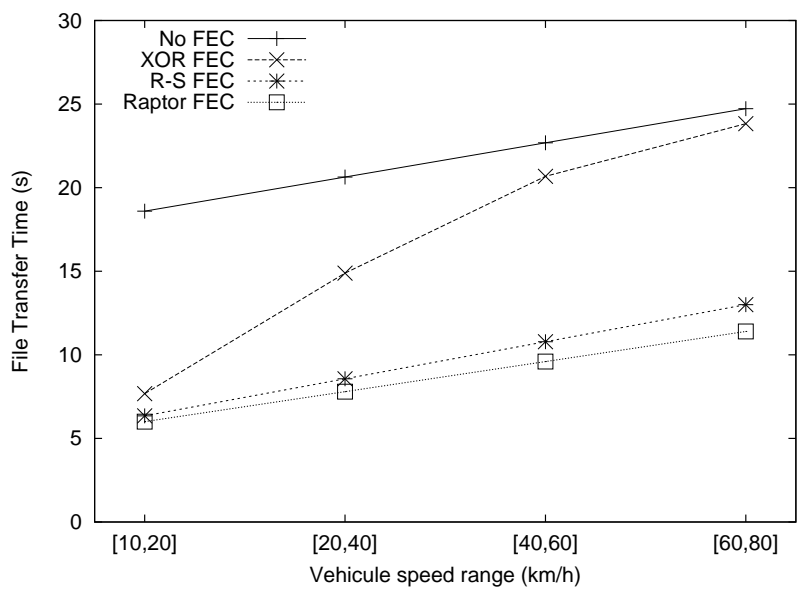

Figure 13: File transfer time for mobile receivers when varying vehicle speed and FEC scheme type.

\section{Acknowledgments}

This work was partially supported by the Ministerio de Educación y Ciencia, Spain, under Grant TIN2008-06441-C02-01.

[1] ITS Handbook Japan, available at: http://www.mlit.go.jp/road/ITS/index/indexHBook.html (2002-2003).

[2] CVIS (Cooperative Vehicle-Infrastructure Systems), available at: http://www.cvisproject.org/en/cvis_project/ (2009).

[3] W.J. Franz, R. Eberhardt, T. Luckenbach, FleetNet-Internet on the road, in: Proc. ITS 2001, Sydney, Australia, 2001.

[4] NOW: Network on Wheels Project, available at www.network-on-wheels.de (2009).

[5] D. Reichardt, M. Miglietta, L. Moretti, P. Morsink, W.Schulz, CarTalk 2000: Safe and comfortable driving based upon inter-vehicle communication, in: Proc. of IEEE Intelligent Vehicle Symposium, 2002, pp. 545-550.

[6] National Highway Traffic Safety Administration, available at: http://www.nhtsa.gov/ (2009).

[7] Fraunhofer Institute for Industrial Engineering, Safespot: cooperative vehicles and road infrastructure for road safety, available at: http://www.safespot-eu.org/ (2009).

[8] IEEE Task group P, IEEE P802.11p: Wireless Access in Vehicular Environments (WAVE), draft standard ed., IEEE Computer Society (2006).

[9] IEEE 802.11 WG, International Standard for Information Technology - Telecom. and Information exchange between systems - Local and Metropolitan Area Networks - Specific Requirements - Part 11: Wireless Medium Access Control (MAC) and Physical Layer (PHY) Specifications, ISO/IEC 8802-11:1999(E) IEEE Std. 802.11 (1999). 
[10] A. Giannoulis, M. Fiore, E. W. Knightly, Supporting vehicular mobility in urban multi-hop wireless networks, in: Proceeding of the 6th international Conference on Mobile Systems, Applications, and Services (MobiSys'08), 2008.

[11] M. Fiore, C. Casetti, C. F. Chiasserini, M. Garetto, Analysis and simulation of a content delivery application for vehicular wireless networks, Elsevier Performance Evaluation 64 (5) (2007) 444-463.

[12] J. Eriksson, H. Balakrishnan, S. Madden, Cabernet: vehicular content delivery using WiFi, in: 14th ACM International Conference on Mobile computing and networking, 2008.

[13] C.-J. Huang, Y.-J. Chen, I.-F. Chen, T.-H. Wu, An intelligent infotainment dissemination scheme for heterogeneous vehicular networks, Elsevier Expert Systems with Applications 2009.

[14] Uichin Lee, Jiyeon Lee, Joon-Sang Park, Mario Gerla, FleaNet: A Virtual Market Place on Vehicular Networks, IEEE Transaction on Vehicular Technology 59 (1) (2010) 344-355.

[15] Y. Sankarasubramaniam, I. F. Akyildiz, S. W. Mclaughlin, Energy efficiency based packet size optimization in wireless sensor networks, in: 1st IEEE International Workshop on Sensor Network Protocols and Applications, Anchorage, Alaska, USA, 2003.

[16] J. Korhonen, Y. Wang, Effect of packet size on loss rate and delay in wireless links, in: IEEE Wireless Communications and Networking Conference, New Orleans, USA, 2005.

[17] P. Lettieri, M. B. Srivastava, Adaptive frame length control for improving wireless link throughput, range, and energy efficiency, in: IEEE INFOCOM, San Francisco, CA, USA, 1998.

[18] Sayantan Choudhury, Jerry Gibson, Payload Length and Rate Adaptation for Throughput Optimization in Wireless LANs, in: 63rd IEEE Vehicular Technology Conference (VTC-Spring), Melbourne, Australia, 2006.

[19] M. Luby, L. Vicisano, Compact Forward Error Correction (FEC) Schemes, RFC 3695 (February 2004).

[20] S. Peltotalo, J. Peltotalo, V. Roca, Simple XOR, Reed-Solomon, and Parity Check Matrixbased FEC Schemes, IETF RMT Working Group, draft-peltotalo-rmt-bb-fec-supp-xor-pcmrs-00.txt (Work in Progress) (June 2004).

[21] J. Lacan, V. Roca, J. Peltotalo, S. Peltotalo, Reed Solomon Error Correction Scheme, IETF RMT Working Group, RFC 5510 ("Standards Track/Proposed Standard") (April 2009).

[22] Amin Shokrollahi, Raptor Codes, IEEE Transactions on Information Theory 52 (6) (2006) 2551-2567.

[23] T. Paila, M. Luby, R. Lehtonen, V. Roca, R. Walsh, FLUTE - File Delivery over Unidirectional Transport, RFC 3926 (October 2004).

[24] M. Luby, J. Gemmell, L. Vicisano, L. Rizzo, J. Crowcroft, Asynchronous Layered Coding (ALC) Protocol Instantiation, IETF RFC 3450 (December 2002).

[25] B. Raman, Channel Allocation in 802.11-Based Mesh Networks, in: IEEE INFOCOM, Barcelona, Spain, 2006. 
[26] MAD Project Flute Implementation, Available at: http://mad.cs.tut.fi/. Accessed: May 2, 2010.

[27] D. Fountain", DF Raptor R11 Encoder/Decoder 2.2.1 Software Development Kit (February 2010).

[28] Moritz Killat, Hannes Hartenstein, An Empirical Model for Probability of Packet Reception in Vehicular Ad Hoc Networks, EURASIP Journal on Wireless Communications and Networking 2009 (Article ID 721301).

[29] K. Fall, K. Varadhan, ns notes and documents., The VINT Project. UC Berkeley, LBL, USC/ISI, and Xerox PARC (February 2000).

[30] M. B. Pursley, D. J. Taipale, Error Probabilities for Spread-Spectrum Packet Radio with Convolutional Codes and Viterbi Decoding, IEEE Transactions on Communications 35 (1).

\section{Appendix A. Derivation of the packet size mapping formula}

In this annex we derive a formula applicable to a Nakagami- $m$ channel that allows calculating the packet arrival/loss ratio when using a certain packet size based on information concerning another packet size.

According to $[18,30]$, we can relate the probability of packet error for a packet size is of $l$ bits when assuming a hard-decision Viterbi decoding at the receiver - $P_{e}^{m}\left(l, \gamma_{s}\right)$ - with the union bound of the first-event error probability corresponding to Nakagami PHY mode $m-P_{u}^{m}\left(\gamma_{s}\right)$ - through equation:

$$
P_{e}^{m}\left(l, \gamma_{s}\right)=1-\left(1-P_{u}^{m}\left(\gamma_{s}\right)\right)^{l}
$$

Lemma 1. If a hard-decision Viterbi decoder can be assumed for the receiver, and if the packet error probability function $P_{e}^{m}\left(l, \gamma_{s}\right)$ is know for a given packet size $l_{\text {ref }}$, the packet arrival probability function for any given packet size $l_{i}$ is:

$$
P_{s}^{m}\left(l_{i}, \gamma_{s}\right)=1-\left[P_{s}^{m}\left(l_{r e f}, \gamma_{s}\right)\right]^{\frac{l_{i}}{l_{r e f}}}
$$

where $P_{s}^{m}\left(l, \gamma_{s}\right)=1-P_{e}^{m}\left(l, \gamma_{s}\right)$.

Proof. First we rearrange equation 11 to make $P_{u}^{m}\left(\gamma_{s}\right)$ the independent member:

$$
\left[1-P_{u}^{m}\left(\gamma_{s}\right)\right]^{l}=1-P_{e}^{m}\left(l, \gamma_{s}\right)
$$

By applying the power of $l^{-1}$ to both sides of the equation we obtain:

$$
P_{u}^{m}\left(\gamma_{s}\right)=1-\left[1-P_{e}^{m}\left(l, \gamma_{s}\right)\right]^{\frac{1}{l}}
$$

Also, since $P_{u}^{m}\left(\gamma_{s}\right)$ is independent of packet size $l$, we can state that:

$$
1-\left[1-P_{e}^{m}\left(l_{1}, \gamma_{s}\right)\right]^{\frac{1}{l_{1}}}=1-\left[1-P_{e}^{m}\left(l_{2}, \gamma_{s}\right)\right]^{\frac{1}{l_{2}}}
$$


where $l_{1}$ and $l_{2}$ are two different packet sizes. We simplify this expression, and then apply the power of $l_{2}$ to both sides of the expression, obtaining:

$$
\left[1-P_{e}^{m}\left(l_{1}, \gamma_{s}\right)\right]^{\frac{l_{2}}{l_{1}}}=1-P_{e}^{m}\left(l_{2}, \gamma_{s}\right)
$$

We are thus able to represent the packet error rate for any packet size $l_{2}$ as a function of the packet error rate for a reference packet length $\left(l_{1}\right)$ :

$$
P_{e}^{m}\left(l_{2}, \gamma_{s}\right)=1-\left[1-P_{e}^{m}\left(l_{1}, \gamma_{s}\right)\right]^{\frac{l_{2}}{l_{1}}}
$$

Since in our case we are interested in the probability of success (arrival) instead, we use the complementary probability equation $P_{s}^{m}\left(l, \gamma_{s}\right)=1-P_{e}^{m}\left(l, \gamma_{s}\right)$ to obtain:

$$
P_{s}^{m}\left(l_{2}, \gamma_{s}\right)=\left[P_{s}^{m}\left(l_{1}, \gamma_{s}\right)\right]^{\frac{l_{2}}{l_{1}}}
$$

